\title{
Central Venous Access Device-Related Bacillus Cereus Endocarditis: A Case Report and Review of the Literature
}

\author{
William F. Wright, DO, MPH, FCAP
}

\begin{abstract}
Bacillus cereus typically presents as a gastrointestinal infection, but rarely manifests as systemic disease. This report describes a case of $B$. cereus-related endocarditis that presented as a sickle cell crisis and bacteremia. Initial clinical suspicion was for laboratory contamination of blood cultures. The case herein described is intended to demonstrate an uncommon presentation of $B$. cereus infection and highlights the value of an aggressive need to further investigate and interpret unexpected blood culture findings in clinical practice, early adequate antimicrobial therapy, prompt diagnosis, and consideration to urgent surgical interventions in such cases.
\end{abstract}

Keywords: Bacillus cereus; Bacillus species; Bacteremia; Central venous catheter infection; Infective endocarditis

$\mathrm{B}$ acillus cereus is a rapidly growing, gram-positive, aerobic-to-facultative, spore forming rod bacteria that exists ubiquitously in the environment, for example, in soil, dust, and plants. ${ }^{1-3}$ The bacterium is also frequently found in food production processes resulting in ingestion of small numbers that participate as part of the transitory human intestinal flora. ${ }^{1}$ Among Bacillus species, Bacillus cereus is the most common in association with gastrointestinal infections as a result of food poisoning. ${ }^{2,3}$ In addition, this species can occasionally cause severe local and systemic infections such as bacteremia, bacterial pneumonia, meningitis, brain abscess, endophthalmitis, ocular keratitis, necrotizing skin and soft-tissue infections, osteomyelitis, pyelonephritis, and endocarditis..$^{1-3}$ Organisms are no longer commonly considered "laboratory contaminants," and an association between Bacillus species and deep-seated infections has been established, especially among immunocompromised patients, those with central venous catheters, and history of intravenous drug use. ${ }^{1-3}$ Endocarditisrelated $B$. cereus infection is a rare clinical presentation, and little is known about its clinical characteristics, treatment, and prognosis. This case represents, to the best of the author's knowledge, the first report of $B$. cereus endocarditis related to a tunneled central venous access device without a history of intravenous drug use. Also presented is a review of the published literature.

\section{Case Presentation}

A man, age 27 years, was admitted to our hospital experiencing generalized joint pains and fevers for approximately 2 weeks. He had no history of orthopedic surgery, joint trauma, or intraarticular injections. He had a history of sickle cell disease classified as sickle cell anemia (homozygous mutation; HbSS) complicated by autosplenectomy and left middle cerebral artery ischemic stroke at age 8 years with residual right upper extremity hemiplegia. The patient has been maintained on disease-modifying therapy with hydroxyurea and folic acid for 19 years. Three years prior to his present admission, he experienced a crisis episode that required exchange transfusion and subsequent placement of a right internal jugular vein tunneled central venous access device (PORT-A-CATH ${ }^{\circledR}$ ) through the anterior chest wall. On physical examination, he was afebrile $\left(99.1^{\circ} \mathrm{F} ; 37.3^{\circ} \mathrm{C}\right)$, and all other vital signs within normal limits except tachycardia (pulse 110 beats per minute). Precordium examination revealed normal first and second heart sounds with a soft, grade $1 / 6$ systolic murmur heard best along the left sternal border and louder with inspiration. Cardiovascular examination did not demonstrate a palpable thrill or elevated jugular venous pressure. His musculoskeletal examination demonstrated no effusions or abnormalities with normal range of motion except flexion contracture formation at the right elbow and generalized muscle wasting of the right

Received:December I, 2015

Ist Revision: January 26, 1016

2nd Revision: February 14, 2016

Accepted: February 19, 2016

doi: $|0.3| 2 \mid / \mathrm{cmr} .2016 .1312$ 
upper extremity. Erythema and warmth were not observed overlying the right chest wall tunneled central venous access device. No skin eruptions, nodules, rash, or nailbed abnormalities were found. Ophthalmologic fundus examination, performed at the bedside, was unremarkable. Pulmonary and abdominal findings were normal.
Laboratory examinations showed that the peripheral white blood cell count was 10,580 cells $/ \mu \mathrm{L}$ (normal range 3,95011,350 cells $/ \mu \mathrm{L}$ ) with neutrophils $62 \%$, lymphocytes $28 \%$, monocytes $6 \%$, and eosinophils $3 \%$. His red blood cell evaluation showed hemoglobin and hematocrit values at $8.0 \mathrm{~g} /$ $\mathrm{dL}$ and $23.4 \%$, respectively, and evidence of hemolysis with

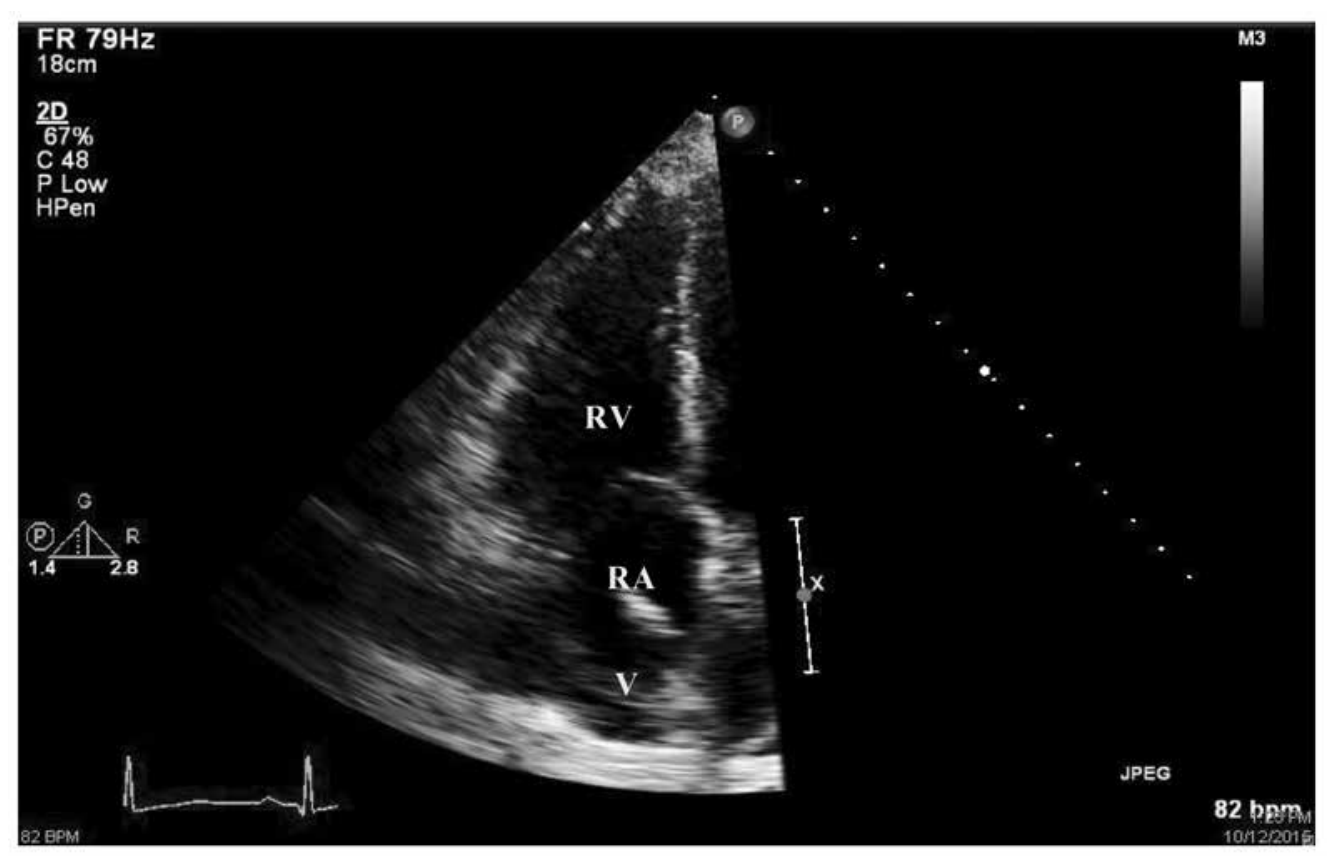

Figure 1. Standard 2-D transthoracic echocardiogram apical four chamber view with focus to the right atrium (RA) and ventricle (RV) demonstrating an ill-defined echogenic dense lesion (V) within the right atrium attached to the Medical Port central venous catheter.

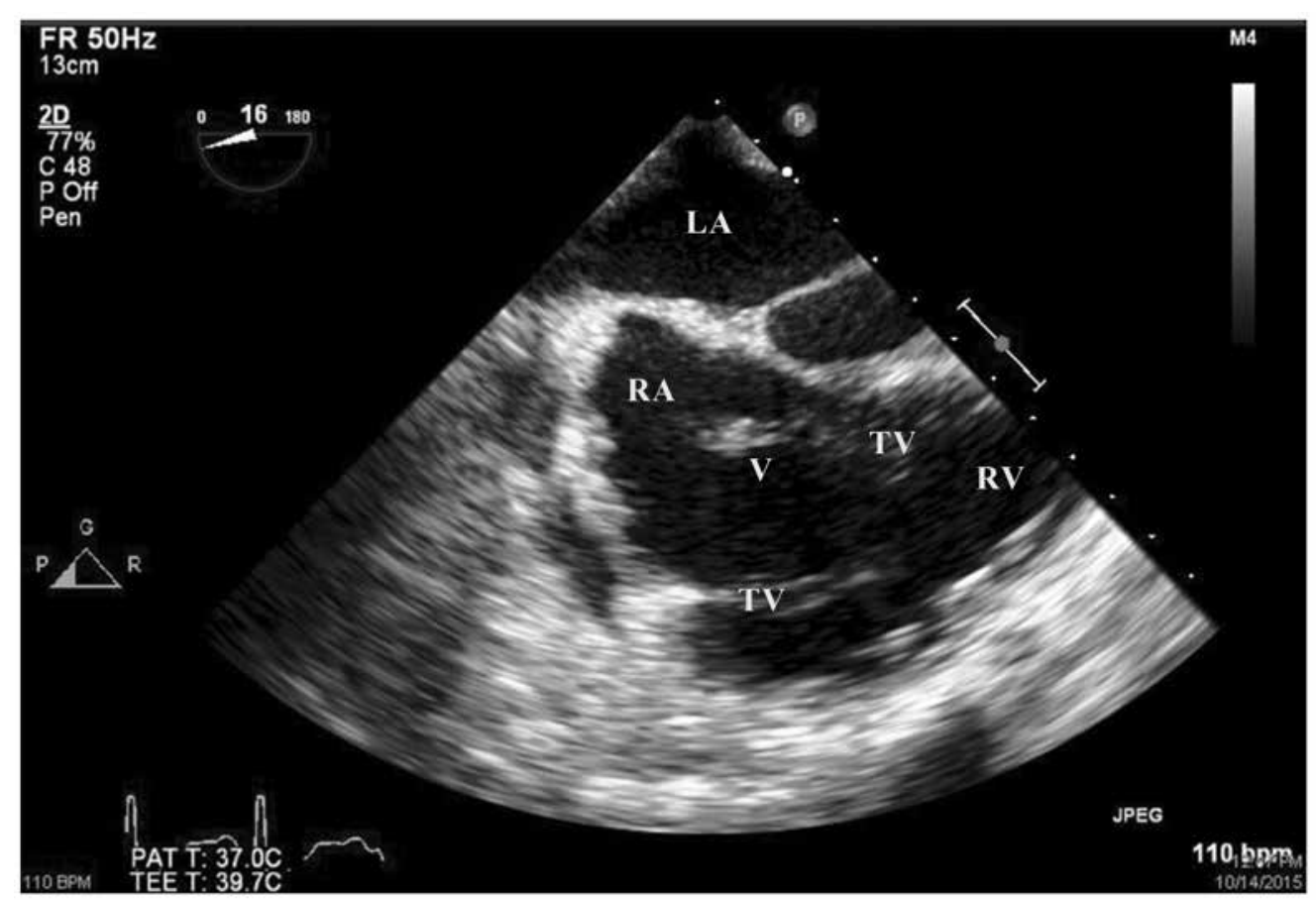

Figure 2. Mid-esophageal transesophageal echocardiogram window (typically at 30-40 cm with an angle range of 0-20 degrees) demonstrating an echogenic dense lesion (V) within the right atrium (RA) attached to the atrial surface of the tricuspid valve (TV). The left atrium (LA) and right ventricle (RV) are also pictured in this view. 
lactate dehydrogenase at $332 \mathrm{U} / \mathrm{L}$ (normal range, 81-234 U/L). Elevated serum levels of C-reactive protein $(7.9 \mathrm{mg} / \mathrm{dL}$, normal range $0.3-0.9 \mathrm{~m} / \mathrm{dL}$ ) and erythrocyte sedimentation rate $(32 \mathrm{~mm} / \mathrm{hr}$, normal range $0-15 \mathrm{~mm} / \mathrm{hr})$ were observed. The serum creatinine was $0.7 \mathrm{mg} / \mathrm{dL}$ (normal range $0.8-$ $1.3 \mathrm{mg} / \mathrm{dL}$ ) with an estimated glomerular filtration ratio of $150 \mathrm{~mL} / \mathrm{min}$. No other abnormalities were detected on laboratory testing including negative blood and urine toxicology screening. Two-view plain-film imaging of the chest revealed no abnormalities. In addition, two sets of blood culture specimens (two aerobic bottles and two anaerobic bottles) from the central venous access device and peripheral veins were inoculated into BACTEC standard culture bottles in a BACTEC 9000 system (Becton, Dickinson and Company, Franklin Lakes, NJ, USA). B. cereus was identified, eventually, upon gram-stain, gross colony morphology, endospore stains, and matrix-assisted laser desorption ionization-time of flight mass spectroscopy (MALDI-TOF MS) protocol, and it grew in all admission blood cultures. Blood cultures through the central venous access port grew within 6 hours, while peripheral blood grew within 10 hours of obtaining samples. Vancomycin 1.25 grams intravenous every 8 hours $(15 \mathrm{mg} / \mathrm{kg}$ dosing protocol) in combination with piperacillin-tazobactam 3.375 grams intravenous every 8 hours treatment was initiated empirically prior to blood culture identification.

Antimicrobial susceptibility was determined with the MicroScan WalkAway system (Dade Behring, Deerfield, IL) and the standard criteria of the Clinical and Laboratory Standards Institute (CLSI). ${ }^{4}$ Breakpoint susceptibility testing was performed using the broth microdilution method with eight antimicrobial agents based upon the recommendations of the CLSI document M24-A2 $\mathrm{E}^{4}$ for the following agents: ciprofloxacin, clindamycin, erythromycin, gentamicin, rifampin, and vancomycin (Table 1). For other antimicrobials (penicillin and tetracycline), the breakpoints for Staphylococcus species in the CLSI guideline M100-S22 ${ }^{5}$ and S246 were used. Based upon culture reporting, the antimicrobial treatment was

Table 1. Minimal inhibitory concentrations for Bacillus cereus isolates

\begin{tabular}{lcc}
\hline Antimicrobial agent & MIC $(\boldsymbol{\mu g} / \mathbf{m L})$ & Susceptibility \\
\hline Ampicillin & $>8$ & $\mathrm{R}$ \\
\hline Ciprofloxacin & $\leq 1$ & $\mathrm{~S}$ \\
\hline Clindamycin & 1 & $\mathrm{I}$ \\
\hline Erythromycin & $\leq 0.2$ & $\mathrm{~S}$ \\
\hline Gentamicin & $\leq 1$ & $\mathrm{~S}$ \\
Rifampin & $\leq 1$ & $\mathrm{~S}$ \\
\hline Tetracycline & $\leq 1$ & $\mathrm{~S}$ \\
Vancomycin & 1 & $\mathrm{~S}$ \\
\hline
\end{tabular}

$\mathrm{MIC}=$ minimum inhibitory concentration

$\mathrm{R}=$ resistance $\mathrm{S}=$ susceptible; $\mathrm{I}=$ intermediate switched to vancomycin monotherapy with subsequent blood cultures becoming negative 1-week later.

The patient's clinical scenario supported the diagnosis of a long-term catheter-related bloodstream infection (CRBSI) according to the published criterion from the Infectious Diseases Society of America (IDSA) for both quantitative blood cultures and differential time to positivity (DTP). ${ }^{7}$ Therefore, the patient underwent further examination by both transthoracic and transesophageal echocardiography. ${ }^{7}$ Twodimensional, M-mode transthoracic echocardiography noted only an ill-defined echogenic density involving the right atrium and indwelling central venous catheter (Figure 1). Transesophageal echocardiography (Figure 2) demonstrated multiple mobile echogenic densities involving the right atrium and tricuspid valve. Tricuspid valvular regurgitation was noted as a new change in comparison to older studies performed at the time of central venous catheter placement and prior stroke. Additionally, contrast-enhanced computed tomography (CT) imaging of the chest demonstrated multiple small pulmonary parenchymal defects consistent with septic emboli. Based on these findings the patient was classified as definite infective endocarditis with two major criteria (eg, oscillating intracardiac and valvular masses as well as new valvular regurgitation) by modification to Duke's criteria for the diagnosis of infective endocarditis. $^{8}$ The patient also met defined criteria for "possible infective endocarditis" with at least one major and one minor manifestation. ${ }^{8}$

Surgical extraction of the tunneled central venous access site was performed followed 72 hours later by placement of a peripherally inserted central venous catheter in the left basilic vein for long-term intravenous antimicrobial therapy. Vancomycin monotherapy was continued for 6 weeks after the first negative set of blood cultures following extraction of the device. Clinically, the patient had cessation of his sickle crisis, resolution of symptoms, normalization of laboratory parameters, and unremarkable end-of-therapy echocardiography examination.

\section{Discussion}

Endocarditis attributed to $B$. cereus and other related species are considered uncommon with few large epidemiological or clinical studies of these types of infections $(1,2,3)$. Therefore, a review of the published literature was performed by conducting a PubMed search of past case reports and case series from 1963 to 2015 with search terms "Bacillus cereus" and "endocarditis;" "Bacillus species" and "endocarditis;" "Bacillus cereus" and "catheter related bloodstream infection;" "Bacillus species" and "catheter related bloodstream infection;" "Bacillus cereus" and "antimicrobial susceptibility;" and "Bacillus species" and "antimicrobial susceptibility;" however, this discussion will mainly focus on B. cereusrelated endocarditis.

Members of the Bacillus cereus group consist of genotypically and phenotypically closely related species to include: $B$. anthracis, B. cereus, B. mycoides, B. pseudomycoides, B. 
thuringiensis, and B. weihenstephanensis. ${ }^{1}$ The most common pathogen implicated in non-anthrax related infections is $B$. cereus. ${ }^{1-3}$ The combination of motility and production of exotoxins, particularly hemolysins (four), phospholipases (three), emetic-toxin (one), and enterotoxins (three) are responsible for the bacteria's ability to cause devastating tissue necrosis in the absence of trauma., ${ }^{1,2}$ Laboratory examination of the bacteria typically reveals a slender, relatively straight or slightly curved, square-end gram-positive rod with an oval centralized endospore. ${ }^{1}$ Bacillus species usually produce good growth on aerobic 5\% sheep blood agar following 24-48 hours of incubation with satisfactory identification using commercial systems such as API Enterobacteriaceae (API 20) and API 50 Carbohydrate (50 $\mathrm{CH})$ kits (bioMerieux, France). ${ }^{1}$ B. cereus is aerotolerant and

Table 2. Summary for cases of Bacillus species endocarditis

\begin{tabular}{|c|c|c|c|c|c|c|c|}
\hline $\begin{array}{l}\text { Case } \\
\text { Year }\end{array}$ & $\begin{array}{l}\text { Bacillus } \\
\text { species }\end{array}$ & $\begin{array}{l}\text { Age (y), } \\
\text { Gender }\end{array}$ & $\begin{array}{l}\text { Risk } \\
\text { factor(s) }\end{array}$ & Valve & $\begin{array}{l}\text { Antimicrobial } \\
\text { therapy [Duration] }\end{array}$ & $\begin{array}{l}\text { Surgical } \\
\text { repair }\end{array}$ & $\begin{array}{l}\text { Clinical } \\
\text { outcome }\end{array}$ \\
\hline $1973^{11}$ & B. subtilis & NR & IVDA & NR & Cfz [NR] & Yes & Survived \\
\hline $1974^{12}$ & B. cereus & $18, F$ & ASD, IVDA & Tricuspid & $\mathrm{Cli} / \mathrm{Lm}[5 \mathrm{wk}]$ & No & Survived \\
\hline $1978^{13}$ & B. cereus & $51, \mathrm{~F}$ & PV & Mitral & Tob/Chl [NR] & No & Deceased \\
\hline $1979^{14}$ & B. cereus & NR & IVDA & NR & Naf $[N R]$ & NR & Survived \\
\hline $1979^{14}$ & B. cereus & NR & IVDA & NR & Cli & NR & Survived \\
\hline $1979^{14}$ & B. cereus & NR & IVDA & NR & Chl, Ery, Gen [NR] & NR & Survived \\
\hline $1979^{15}$ & B. cereus & NR & RHD & Aortic & Pen, Gen, Str [NR] & No & Deceased \\
\hline $1979^{16}$ & B. cereus & $50, F$ & IVDA & None & Cli, Kan [4 wk] & No & Survived \\
\hline $1982^{17}$ & B. cereus & $55, M$ & $\begin{array}{l}\text { PV } \\
\text { (porcine) }\end{array}$ & Aortic & Cli, Gen [6 wk] & Yes & Survived \\
\hline $1987^{18}$ & B. cereus & $43, F$ & PM, RHD & PM Wire & Cli [6 wk] & Yes & Survived \\
\hline $1991^{19}$ & B. circulans & $N R$ & $A A, V H D$ & Aortic & Pen, Tob [NR] & Yes & Survived \\
\hline $1992^{20}$ & B. cereus & $34, \mathrm{M}$ & PV & Aortic & Van [6 wk] & Yes & Survived \\
\hline $1994^{21}$ & B. cereus & $43, M$ & PV & Mitral & Amk, Min [NR] & Yes & Survived \\
\hline $1995^{22}$ & B. licheniformis & $73, \mathrm{M}$ & PV, VHD & Aortic & $\mathrm{Cfz}[6 \mathrm{wk}]$ & Yes & Survived \\
\hline $1998^{23}$ & B. cereus & $\mathrm{NR}$ & PV & Mitral & Gen, Rif, Van [NR] & Yes & Survived \\
\hline $1999^{24}$ & B. cereus & $45, \mathrm{~F}$ & PV & Mitral & Gen, Rif, Van [6 wk] & Yes & Survived \\
\hline $1999^{25}$ & B. circulans & $56, F$ & $\mathrm{PV}, \mathrm{RHD}$ & Aortic & Cip, Sxt [6 wk] & Yes & Survived \\
\hline $1999^{26}$ & B. popilliae & $57, \mathrm{M}$ & BAV & Aortic & Pen [6 wk] & No & Survived \\
\hline $2003^{27}$ & B. pumilus & $42, M$ & PFO, PV & PV & Van [6 wk] & No & Survived \\
\hline $2005^{28}$ & B. cereus & $38, M$ & ALL & Mitral & Pen, Van [3 wk] & No & Deceased \\
\hline $2007^{29}$ & Bacillus sp. & $68, M$ & ICD & Wire & Van $[6 \mathrm{wk}]$ & No & Survived \\
\hline $2008^{30}$ & B. cereus & $69, \mathrm{~F}$ & PM & Wire & Cfz [6 wk] & No & Survived \\
\hline $2011^{31}$ & B. cereus & $42, M$ & None & Aortic & Cro [6 wk] & Yes & Survived \\
\hline $2012^{32}$ & B. cereus & $54, \mathrm{M}$ & VHD & Mitral & NR [6 wk] & Yes & Survived \\
\hline $2013^{33}$ & B. cereus & $65, M$ & PM & Wire & Ofx, Pip [4 wk] & Yes & Deceased \\
\hline $2015^{34}$ & B. cereus & $31, \mathrm{M}$ & IVDA & Aortic & Cxm [6 weeks] & No & Survived \\
\hline $2015^{35}$ & B. cereus & $30, \mathrm{~F}$ & IVDA, *Prg & Tricuspid & Van $[6 \mathrm{wk}]$ & No & Survived \\
\hline $\begin{array}{l}\text { Current } \\
\text { case }\end{array}$ & B. cereus & $27, M$ & MP-CVC & Tricuspid & Van [6 wk] & Yes & Survived \\
\hline
\end{tabular}

$N R$, not reported; $F$, female; $M$, male

AA, alcohol abuse; ALL, acute lymphoblastic leukemia; ASD, atrial septal defect; BAV, bicuspid aortic valve; ICD, implantable cardioverter defibrillator; IVDA, intravenous drug abuse; MP-CVC, medical port central venous catheter; PFO, patent foramen ovale; PM, pacemaker; PV, prosthetic valve; RHD, rheumatic heart disease; VHD, valvular heart disease

Amk, amikacin; Cfz, cefazolin; Chl, chloramphenicol; Cip, ciprofloxacin; Cli, clindamycin; Cro, ceftriaxone; Cxm, cefuroxime; Ery, erythromycin; Gen, gentamicin; Kan, kanamycin; Lm, lincomycin; Min, minocycline; Naf, nafcillin; Pen, penicillin; Rif, rifampin; Str, streptomycin; Sxt, trimethoprim-sulfamethoxazole; Tob, tobramycin; Van, vancomycin

*Prg, the patient was 25 -weeks pregnant at the time of presentation with normal delivery at 37 weeks. 
grows readily as swarming, flat, dull gray, or opaque colonies with a narrow zone of $\beta$-hemolysis on aerobic blood agar. ${ }^{1,2}$

Recognition of systemic infection caused by non-anthrax Bacillus species began in 1963 with Farrar's review of 12 cases reported in the world literature. ${ }^{9}$ Finland and Barnes ${ }^{10}$ in their review first reported three cases (two cases in 1933 and one case in 1951) with gram-positive bacillus (not specifically reported as organisms of the genus Bacillus) endocarditis at Boston City Hospital. Endocarditis caused by nonpathogenic organisms of the genus Bacillus (B. subtilis) was first reported in 1973 with an intravenous drug-abusing (IVDA) patient. ${ }^{11}$ Subsequently, B. cereus endocarditis was first reported in 1974 with a young female heroin addict. ${ }^{12}$ Since then, 26 additional cases of Bacillus species-related endocarditis have been reported in the literature (Table 2), ${ }^{13-35}$ While B. cereus is the most commonly reported pathogen $(78.5 \% ; n=22)$, other associated species include: $B$. circulans (two cases), $B$. licheniformis (one case), B. popilliae (one case), B. pumilus, and $B$. subtilis (one case). Males are reported more commonly than females $(42.8 \%$ vs $25.0 \%)$, with age distributions of $10-21$ years $(3.6 \% ; n=1), 21-40$ years $(14.2 \% ; n=4), 41-60$ years $(35.7 \% ; n=10)$, and over 60 years $(14.2 \% ; n=4)$.

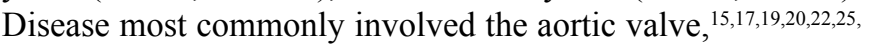
${ }^{26,31,34}$ followed by the mitral, ${ }^{13,21,23,24,28}$ and tricuspid valve, ${ }^{12,35}$ respectively. When Bacillus species are found to be the causative pathogen of endocarditis they are typically associated with IVDA history, ${ }^{11,12,14,16,34,35}$ leukemia, ${ }^{28}$ pre-existing cardiac or valvular heart disease (atrial septal defect; bicuspid aortic valve; patent foramen ovale; rheumatic heart disease), ${ }^{12,18,19,22,}$ 25-27,32 prosthetic heart valve, ${ }^{13,17,20-25,27}$ pacemaker/implantable cardioverter defibrillator, ${ }^{18,29,30,33}$ and pregnancy. ${ }^{35}$ This report describes the $29^{\text {th }}$ overall case of Bacillus species-related endocarditis, the $22^{\text {nd }}$ involving $B$. cereus, the third tricuspid valve disease, and the first involving sickle cell disease with a tunneled medical port central venous catheter in a patient without IVDA.

Previous reports suggest IVDA $^{11,12,14,16,34-36}$ and in-dwelling central venous catheters ${ }^{37-40}$ as risk factors most commonly associated with Bacillus species-related bacteremia. Bacteremia most likely originates from cutaneous colonization and/or contamination of drug agents and injection equipment. ${ }^{31,35-40}$ Tuazon et $\mathrm{al}^{36}{ }^{36}$ when comparing bacterial cultures among 100 samples each of heroin and injection paraphernalia, found Bacillus species could be cultured from $32 \%$ and $47 \%$, respectively. In a retrospective evaluation of 94 adult patients with Bacillus bloodstream infection (2,054 positive blood cultures) at a tertiary medical center between 1990 and 2008, Kassar et $\mathrm{al}^{40}$ defined more than half (71\%) the cases as probable CRBSI followed by definite CRBSI in $28 \%$ of cases. The association between Bacillus species and intravascular devices results mainly from high exopolymer production (biofilm formation). ${ }^{40}$ Other studies have demonstrated the presence of $B$. cereus within ethanol containing preparations as well as hospital linens and towels in association with nosocomial-related bacteremia. ${ }^{41-43}$
Most of the patients listed in Table 2, including this case, did not have IVDA history-related Bacillus species bloodstream infection. While IVDA-associated prosthetic valve infection responded to valve replacement and systemic antimicrobial therapy, native valve IVDA-associated infections typically responded to antimicrobial therapy alone. In this case, the presence of a long-term central venous catheter provides the most probable explanation of how the organism gained access to cause infection; however, the role of many previous hospitalizations for sickle cell crisis cannot be ruled out in this case. Previous studies have reported resolution of Bacillus species-related CRBSI with central venous catheter removal, ${ }^{38-40}$ and therefore, this patient was treated with both catheter removal and systemic antimicrobial therapy.

The patient under discussion was eventually diagnosed with B. cereus-related endocarditis based on admission blood cultures, evidence supporting long-term CRBSI and echocardiographic findings. This case was classified as both "possible infective endocarditis" with at least one major and one minor manifestation and "definite infective endocarditis" with two major criteria (eg, oscillating intracardiac and valvular masses as well as new valvular regurgitation) by modifications to Duke's criteria for the diagnosis of infective endocarditis. ${ }^{8}$ New tricuspid valvular regurgitation was based on prior normal transthoracic echocardiogram reports at the time of central venous catheter placement and stroke. While the majority of patients listed in Table 2 fulfilled the criterion of possible infective endocarditis with one major manifestation (positive echocardiogram) and minor manifestation (predisposing heart condition or injection drug use), it is unclear from the available data if cases met the strict criterion of definite infective endocarditis (eg, two major criteria; one major criterion and three minor criteria; or five minor criteria). ${ }^{8}$

Regarding treatment, patients listed in Table 2 exhibited varying antimicrobial regimens for, most commonly, a duration of 6 weeks. According to antimicrobial susceptibility studies, B. cereus appears to be uniformly sensitive to gentamicin, imipenem, and vancomycin. ${ }^{44-48}$ In general, three separate trials by Weber et al, ${ }^{44}$ Luna et al, ${ }^{46}$ and Veysseyre et $\mathrm{al}^{48}$ report all tested strains $(100 \% ; \mathrm{n}=240)$ susceptible to gentamicin, imipenem, and vancomycin. Most strains were variably resistant to amoxicillin (40\%), cefazolin $(55 \%)$, ceftriaxone $(40 \%)$, ciprofloxacin $(41 \%)$, clindamycin $(20 \%)$, and penicillin $(100 \%) \cdot{ }^{44,46,48}$ Luna et $\mathrm{al}^{46}$ reported $42 \mathrm{~B}$. cereus isolates that all were susceptible to daptomycin, linezolid, and tigecycline. Ikeda et $\mathrm{al}^{47}$ reported a retrospective single-center trial involving 29 evaluable patients to document the clinical and antimicrobial susceptibility characteristics of $B$. cereus nosocomial bloodstream infections as well as whether appropriate empirical treatment $(n=9)$ or inappropriate empirical treatment $(n=20)$ had been initiated at admission (defined as susceptible antimicrobial agents). No significant 
difference existed in the clinical responses of the two groups with all-cause mortality, but early defervescence occurred more often with appropriate empirical therapy than with inappropriate empirical antimicrobial therapy. Authors reported $65.5 \%$ of isolates resistant to clindamycin and $10.3 \%$ resistant to levofloxacin. Empirical antimicrobial therapy is often initiated prior to culture at the time of evaluation with emergency medicine providers, followed by modification with susceptibility data; therefore, the most appropriate empirical choices include daptomycin, imipenem, linezolid, tigecycline, or vancomycin. Although the patient reported in this current case was empirically treated with vancomycin 1.25 grams intravenous every 8 hours $(15 \mathrm{mg} / \mathrm{kg})$ in combination with piperacillin-tazobactam 3.375 grams intravenous every 8 hours, his strain demonstrated resistance only to ampicillin and intermediate susceptibility to clindamycin (Table 1). Based on susceptibility testing, vancomycin monotherapy was continued for 6 weeks after the first negative set of blood cultures following extraction of the device. Clinically, the patient had cessation of his sickle crisis, resolution of crisis-related symptoms, normalization of laboratory parameters, and unremarkable end-of-therapy transthoracic echocardiography examination with mild residual tricuspid regurgitation but complete resolution of valvular vegetation.

Although this patient was reported as afebrile with a normal peripheral leukocyte count, it is not clear whether this represents a typical or atypical clinical presentation, because of the lack of data and varying reports with previous cases. In contrast, Kassar et a ${ }^{40}$ reported $88 \%$ of patients ( 83 of 93 ) had a fever (defined as greater than $38.0^{\circ} \mathrm{C}$ ) among 94 episodes of Bacillus species-related bloodstream infections. Additionally, Ikeda et $\mathrm{al}^{47}$ reported an average white blood cell count of 7,497 cells $\mu \mathrm{L}$ (reported range of $100-22,200$ cells $/ \mu \mathrm{L}$ ) among 29 patients with B. cereus-related bloodstream infections. Therefore, further evaluations are needed to elucidate clinical characteristics for patients with Bacillus species-related endocarditis.

\section{Conclusion}

This report describes a case of the first patient with B. cereusrelated endocarditis in association with sickle cell disease and a medical port central venous catheter, and includes a literature review. Given that Bacillus species-related endocarditis is uncommon, this case highlights an aggressive need to further investigate and interpret unexpected blood culture findings in clinical practice. Another important lesson from this case report and literature review would suggest early, adequate antimicrobial therapy, prompt diagnosis, and consideration to urgent surgical interventions with either valve replacement or device extraction. Finally, recognition of infections due to unusual pathogens warrants further evaluation and appropriate management in association with an infectious disease consultation.

\section{References}

1. Bottone EJ. Bacillus cereus, a volatile human pathogen. Clin Microbiol Rev 2010;23:382398.

2. Drobniewski FA. Bacillus cereus and related species. Clin Microbiol Rev 1993;6:324-338.

3. Ihde DC, Armstrong D. Clinical spectrum of infection due to Bacillus species. Am J Med 1973;55:839-845.

4. Clinical and Laboratory Standards Institute (CLSI). Methods for antimicrobial dilution and disk susceptibility testing of infrequently isolated or fastidious bacteria; approved guideline, $2^{\text {nd }}$ edn. M45A2E. Wayne, PA: CLSI; 2010.

5. Clinical and Laboratory Standards Institute (CLSI). Performance standards for antimicrobial susceptibility testing; twentysecond information supplement M100-S22. Wayne, PA: CLSI; 2012.

6. Clinical and Laboratory Standards Institute (CLSI). Performance standards for antimicrobial susceptibility testing; twentyfourth information supplement M100-S24. Wayne, PA: CLSI; 2014.

7. Mermel LA, Allon M, Bouza E, Craven DE, Flynn P, O'Grady NP, Raad II, Rijnders BJ, Sherertz RJ, Warren DK. Clinical practice guidelines for the diagnosis and management of intravascular catheter-related infection: 2009 Update by the Infectious Diseases Society of America. Clin Infect Dis 2009;49:1-45

8. Li JS, Sexton DJ, Mick N, Nettles R, Fowler VG Jr, Ryan T, Bashore T, Corey GR. Proposed modifications to the Duke criteria for the diagnosis of infective endocarditis. Clin Infect Dis 2000;30:633-638.

9. Farrar WE Jr. Serious infections due to "non-pathogenic" organisms of the genus bacillus. Am J Med 1963;34:134-141.

10. Finland M, Barnes MW. Changing etiology of bacterial endocarditis in the antibacterial era. Experiences at Boston City Hospital 1933-1965. Ann Intern Med 1970;72:341-348.

11. Reller LB. Endocarditis caused by Bacillus subtilis. Am J Clin Pathol 1973;60:714-718

12. Craig CP, Lee WS, Ho M. Letter: Bacillus cereus endocarditis in an addict. Ann Intern Med 1974;80:418-419.

13. Block CS, Levy ML, Fritz VU. Bacillus cereus endocarditis. A case report. S Afr Med J 1978;53:556-557.

14. Tuazon CU, Murray HW, Levy C, Solny MN, Curtin JA, Sheagren JN. Serious infections from Bacillus sp. JAMA. 1979;241:1137-1140.

15. Wanvarie S, Rochanawatanon M. Bacillus cereus endocarditis. J Med Assoc Thai 1979;62:34-38

16. Weller PF, Nicholson A, Braslow N. The spectrum of Bacillus bacteremias in heroin addicts. Arch Intern Med 1979;139:293-294.

17. Oster HA, Kong TQ. Bacillus cereus endocarditis involving a prosthetic valve. South Med J 1982;75:508-509.

18. Sliman R, Rehm S, Shlaes DM. Serious infections caused by Bacillus species. Medicine (Baltimore) 1987;66:218-223.

19. Gatermann S, Mitusch R, Djonalgic H, Hollandt H, Marre R. Endocarditis caused by Bacillus circulans. Infection 1991;19:445.

20. Steen MK, Bruno-Murtha LA, Chaux G, Lazar H, Bernard S, Sulis C. Bacillus cereus endocarditis: report of a case and review. Clin Infect Dis 1992;14:945-946.

21. Yamamura M, Aoki K, Takanashi S, Tadokoro M, Furuta S, Mizokami T. [A case of Bacillus cereus prosthetic valve endocarditis]. [Article in Japanese] Kyobu Geka. 1994:47:232-234

22. Santini F, Borghetti V, Amalfitano G, Mazzucco A. Bacillus licheniformis prosthetic aortic valve endocarditis. J Clin Microbiol 1995;33:3070-3073. 
23. Martín Cadenas P, Sánchez Alor G, Aguilar Ruiz JC, Castedo E, Daza R, Mendaza P. [Endocarditis by Bacillus cereus 1 in prosthetic mitral valve]. [Article in Spanish] Enferm Infecc Microbiol Clin 1998;16:102-104.

24. Castedo E, Castro A, Martin P, Roda J, Montero CG. Bacillus cereus prosthetic valve endocarditis. Ann Thorac Surg 1999; 68:2351-2352.

25. Krause A, Gould FK, Forty J. Prosthetic heart valve endocarditis caused by Bacillus circulans. J Infect 1999;39:160-162.

26. Wu YJ, Hong TC, Hou CJY, Chou YS, Cheng-Ho T, Yang DI. Bacillus popilliae endocarditis with prolonged complete heart block. Am J Med Sci 1999;317:263-265.

27. Apisarnthanarak A, Little JR, Stoner BP. Endocarditis due to Bacillus pumilus: the significance of non-cereus, nonanthracis Bacillus. Journal of Infectious Diseases and Antimicrobial Agents 2003;20(3):155-157. Available at: http://www.idthai.org/Publication/pdf/Vol20-3.pdf.f

28. Cone LA, Dreisbach L, Potts BE, Comess BE, Burleigh WA. Fatal Bacillus cereus endocarditis masquerading as an anthrax-like infection in a patient with acute lymphoblastic leukemia: case report. J Heart Valve Dis. 2005;14:37-39.

29. Shalev A, Gilad J, Riesenberg K, Borer A, Kobal S, Schlaeffer F, Katz A. Conservative management of implantable cardioverter defibrillator-related endocarditis due to Bacillus spp. Infection 2007;35:114-117.

30. Abusin S, Bhimaraj A, Khadra S. Bacillus Cereus Endocarditis in a permanent pacemaker: a case report. Cases J. 2008;1:95.

31. Thomas BS, Bankowski MJ, Lau WK. Native valve Bacillus cereus endocarditis in a non-intravenous-drug-abusing patient. J Clin Microbiol. 2012;50:519-521.

32. Oh DH, Kim MH, Kim YC, Song JE, Ahn JY, Han SH, Choi JY, Kim CO, Chang HJ, Yong D, Song YG, Lee Y, Kim JM. A case of native valve infective endocarditis caused by Bacillus cereus. Infection \& Chemotherapy. 2012;44(4):310-314. Available at: http://synapse.koreamed. org/DOIx.php?id=10.3947/ ic. 2012.44.4.310\&vmode=PUBREADER.

33. Barraud O, Hidri N, Ly K, Pichon N, Manea P, Ploy MC, Garnier F. Pacemaker-associated Bacillus cereus endocarditis. Diagn Microbiol Infect Dis 2012;74:313-315.

34. Ngow HA, Wan Khairina WM. Bacillus cereus endocarditis in native aortic valve. J Infect Chemother 2013;19:154-157.

35. Shah M, Patnaik S, Wongrakpanich S, Alhamshari Y, Alnabelsi T. Infective endocarditis due to Bacillus cereus in a pregnant female: A case report and literature review. IDCases 2015;2:120-123.

36. Tuazon CU, Hill R, Sheagren JN. Microbiologic study of street heroin and injection paraphernalia. J Infect Dis 1974; 129:327-329.

37. Blue SR, Singh VR, Saubolle MA. Bacillus licheniformis bacteremia: five cases associated with indwelling central venous catheters. Clin Infect Dis 1995;20:629-633.

38. Koch A, Arvand M. Recurrent bacteraemia by 2 different Bacillus cereus strains related to 2 distinct central venous catheters. Scand J Infect Dis 2005;37:772-774.

39. Bentur HN, Dalzell AM, Riordan FA. Central venous catheter infection with Bacillus pumilus in an immunocompetent child: a case report. Ann Clin Microbiol Antimicrob 2007;6:12.

40. Kassar R, Hachem R, Jiang Y, Chaftari AM, Raad I. Management of Bacillus bacteremia: the need for catheter removal. Medicine (Baltimore) 2009;88:279-283.

41. Barrie D, Hoffman PN, Wilson JA, Kramer JM. Contamination of hospital linen by Bacillus cereus. Epidemiol Infect 1994;113:297-306.
42. Dohmae S, Okubo T, Higuchi W, Takano T, Isobe H, Baranovich T, Kobayashi S, Uchiyama M, Tanabe Y, Itoh M, Yamamoto T. Bacillus cereus nosocomial infection from reused towels in Japan. J Hosp Infect. 2008;69:361-367.

43. Sasahara T, Hayashi S, Morisawa Y, Sakihama T, Yoshimura A, Hirai Y. Bacillus cereus bacteremia outbreak due to contaminated hospital linens. Eur J Clin Microbiol Infect Dis 2011;30:219-226.

44. Weber DJ, Saviteer SM, Rutala WA, Thomann CA. In vitro susceptibility of Bacillus spp. to selected antimicrobial agents. Antimicrob Agents Chemother 1988;32:642-645.

45. Turnbull PCB, Sirianni NM, LeBron CI, Samaan MN, Sutton FN, Reyes AE, Peruski LF Jr. MICs of selected antibiotics for Bacillus anthracis, Bacillus cereus, Bacillus thuringiensis, and Bacillus mycoides from a range of clinical and environmental sources as determined by the Etest. J Clin Microbiol 2004;42:3626-3634.

46. Luna VA, King DS, Gulledge J, Cannons AC, Amuso PT, Cattani J. Susceptibility of Bacillus anthracis, Bacillus cereus, Bacillus mycoides, Bacillus pseudomycoides and Bacillus thuringiensis to 24 antimicrobials using Sensititre automated microbroth dilution and Etest agar gradient diffusion methods. J Antimicrob Chemother 2007;60:555-567.

47. Ikeda M, Yagihara Y, Tatsuno K, Okazaki M, Okugawa S, Moriya K. Clinical characteristics and antimicrobial susceptibility of Bacillus cereus blood stream infections. Ann Clin Microbiol Antimicrob 2015;14:43.

48. Veysseyre F, Fourcade C, Lavigne JP, Sotto A. Bacillus cereus infection: 57 case patients and a literature review. Med Mal Infect 2015;45:436-440.

\section{Author Affiliation}

William F. Wright, DO, MPH, FCAP; Division of Infectious Diseases, Department of Medicine, Memorial Medical Center, York, Pennsylvania, USA 